Geosciences Journal

Vol. 17, No. 3, p. 387 - 387, September 2013

DOI 10.1007/s12303-013-0042-y

(C) The Association of Korean Geoscience Societies and Springer 2013

\title{
Erratum to: Possible origin of coastal sands and their long-term distribution along the high slope-gradient, wave-dominated eastern coast, Korea
}

\author{
Sang Hoon Lee* \\ Hee Jun Lee \\ Jun-Yong Park \\ Korea Institute of Ocean Science and Technology, Ansan, 425-600, Republic of Korea \\ Eui Young Jeong \\ Jeseon Yoo \\ Ho Kyung $\mathrm{Ha}$ \\ Korea Polar Research Institute, Incheon 406-840, Republic of Korea \\ Chang Woong Shin \\ Chanhong Park \\ Korea Institute of Ocean Science and Technology, Ansan, 425-600, Republic of Korea
}

Erratum to: Geosciences Journal, 2013, 17, 163-172

DOI 10.1007/s12303-013-0010-6

One of the authors' names was misprinted. The author list

should be corrected as follows.

Sang Hoon Lee*, Hee Jun Lee, Jun-Yong Park, Eui Young Jeong, Jeseon Yoon, Ho Kyung Ha, Chang Woong Shin, and Chanhong Park

\section{Corrected Author list}

Sang Hoon Lee*, Hee Jun Lee, Jun-Yong Park, Eui Young Jeong, Jeseon Yoo, Ho Kyung Ha, Chang Woong Shin, and Chanhong Park

The online version of the original article can be found under DOI 10.1007/s12303-013-0010-6

*Corresponding author: sanglee@kordi.re.kr 